\title{
Computed tomography and magnetic resonance imaging features of ovarian fibrothecoma
}

\author{
JINGYA CHEN $^{1}$, JIANHUA WANG ${ }^{1}$, XIAO CHEN $^{1}$, YAOHUI WANG $^{2}$, ZHONGQIU WANG $^{1}$ and DAKE LI $^{3}$ \\ Departments of ${ }^{1}$ Radiology, ${ }^{2}$ Pathology and ${ }^{3}$ Gynecology, Affiliated Hospital of Nanjing University of Chinese Medicine, \\ Nanjing, Jiangsu 210029, P.R. China
}

Received June 15, 2016; Accepted March 17, 2017

DOI: $10.3892 / 01.2017 .6228$

\begin{abstract}
The aim of the present study was to investigate the imaging characteristics of ovarian fibrothecoma. The cases of 25 female patients with pathologically confirmed ovarian fibrothecomas were retrospectively reviewed. Tumor location, size, density, signal intensity, cystic degeneration, calcification, enhancement pattern and ascites were assessed by computed tomography $(\mathrm{CT})(\mathrm{n}=20)$ and magnetic resonance imaging (MRI) (n=5). Clinical and histological features were also evaluated. The results revealed that $23(92.0 \%)$ patients were postmenopausal, with a mean age \pm standard deviation (SD) of $60.7 \pm 10.8$ years old. All 25 tumors were unilateral and a thickened endometrium was observed in $7(28.0 \%)$ patients. In total, $24(96.0 \%)$ tumors appeared to have well-defined boundaries, with $1(4 \%)$ tumor exhibiting an obscure boundary. Tumors had a mean size of $9.8 \pm 5.3 \mathrm{~cm}$. Solid and predominantly solid masses were found in 16 cases (64.0\%), and cystic and predominantly cystic masses were found in 9 patients (36.0\%). Solid regions of masses exhibited hypodensity or isodensity in 24 patients $(96.0 \%)$, with 1 patient $(4.0 \%)$ exhibiting hyperdensity. The mean CT value $( \pm \mathrm{SD})$ was $44.2 \pm 12.8 \mathrm{HU}$ (range, 31-79HU) in unenhanced images. All masses exhibited isointensity or slight hypointensity on T1-weighted imaging, and tumor parenchyma also exhibited slight hypointensity or isointensity on T2-weighted imaging compared with the myometrium, with or without areas of patchy hyperintensity. Contrast-enhanced CT or MRI scans were performed on 19 patients, and the solid components (94.7\%) exhibited mild to moderate enhancement in 18 patients and 1 patient $(5.3 \%)$
\end{abstract}

Correspondence to: Professor Zhongqiu Wang, Department of Radiology, Affiliated Hospital of Nanjing University of Chinese Medicine, 155 Hanzhong Road, Nanjing, Jiangsu 210029, P.R. China E-mail: zhq2001us@163.com

Professor Dake Li, Department of Gynecology, Affiliated Hospital of Nanjing University of Chinese Medicine, 155 Hanzhong Road, Nanjing, Jiangsu 210029, P.R. China

E-mail: lidake2002@163.com

Key words: ovary, fibrothecoma, computed tomography, magnetic resonance imaging exhibited intense enhancement. Tumor size was positively correlated with the tumor cystic degeneration $(r=0.77, \mathrm{P}<0.001)$ and ascites $(r=0.41, P<0.001)$. Therefore, ovarian fibrothecoma typically presents as solid or predominant solid masses with clear boundaries; the parenchyma of the tumor exhibits isodensity on CT scans, slight hypointensity or isointensity on MRI and mild enhancement following contrast-medium injection. The thickened endometrium observed in postmenopausal woman may also be a valuable imaging feature.

\section{Introduction}

Ovarian fibrothecoma is an uncommon type of ovarian tumor of the thecoma-fibroma group, which is a set of tumors of sex cord/stromal origin. The thecoma-fibroma group is a spectrum of benign ovarian tumors that contains fibroma, fibroma-thecoma and thecoma, depending on the relative proportion of fibroblasts and theca cells (1).

Fibromas are composed entirely or almost entirely of fibroblasts and no theca cells, whereas fibroma-thecoma and thecoma are composed of different proportion of theca cells. Fibromas and fibrothecomas are considered to be the most common solid primary tumors of the ovary (2). Fibroma-thecomas and thecomas share overlapping histological characteristics (1), so the designation of fibrothecoma is used. This tumor accounts for $\sim 4 \%$ of all ovarian neoplasms (1). Due to the low prevalence, the imaging features of fibrothecoma are not fully known, which results in easy misdiagnosis (3).

Fibrothecomas originate from the ovarian medulla, whereas fibromas originate from the ovarian cortex (2). The difference in origin and cellular components leads to different clinical and imaging manifestations (3). Fibromas are primarily observed in young women and are usually non-functioning tumors. Fibrothecomas, however, are usually observed in postmenopausal women; the mean age of onset is between 55 and 60 years (4). Fibrothecomas are the most common hormonally active ovarian neoplasms (5). Almost half produce estrogen. A high level of estrogen, menstrual disorders and vaginal bleeding are occasionally observed in patients with fibrothecoma (1). They are considered to be benign; malignancy in fibrothecomas is rarely observed (6).

To the best of our knowledge, several studies have previously described the imaging features of ovarian fibroma and 
fibrothecoma $(1,4,5,7)$. However, the population sample sizes were small and the different disease subtypes were always discussed together. Nocito et al (8) indicated that fibroma and fibrothecoma should be considered as separate entities. $\mathrm{Wu}$ et al (7) previously reported on the magnetic resonance imaging (MRI) features of fibrothecoma in 26 patients, and showed that MRI had the superiority to show the ovarian fibrothecoma characteristics as well as other oestrogenic functional appearances. The present study retrospectively analyzed the computed tomography (CT) and MRI features of 25 histologically-proven fibrothecomas. Histological features were also discussed and compared with the imaging characteristics.

\section{Patients and methods}

Patients. The present retrospective study was approved by the Institutional Review Board of the Affiliated Hospital of Nanjing University of Chinese Medicine and requirement of written informed consent was waived. Twenty-six patients with surgically and pathologically proven ovarian fibrothecomas treated at the Affiliated Hospital of Nanjing University of Chinese Medicine between January 2009 and February 2015 were included. Of the 26 patients, 1 was excluded as their tumor was smaller than $1 \mathrm{~cm}$, which was almost too small to be measured; the remaining 25 cases were analyzed.

CT and MRI scan protocol. A total of 20 patients were examined with a 64-row multislice-spiral CT (Brilliance 64; Philips Healthcare, DA Best, The Netherlands). Of these 20 patients, 15 underwent contrast-enhanced CT scans with the intravenous administration of Ultravist. The scanning protocol was as follows: 5-mm layer thickness, a pitch of $1,120-\mathrm{kV}$ tube voltage and 200-500-mA tube current. CT images were processed in workstation for coronal, sagittal and oblique reconstruction.

A total of 5 patients underwent a 3.0T (Verio, Siemens AG, Munich, Germany) MRI examination using a body-phase array coil. All sequences were acquired with anterior and posterior saturation bands. The transverse planes covered the entire area of the pelvis. Axial fast low-angle shot two-dimensional T1-weighted image (T1WI) [time of reception (TR)/time of echo (TE), 139/4.76 msec; thickness, $6.0 \mathrm{~mm}$; interaction gap, $1 \mathrm{~mm}$; field of view, $25 \mathrm{~cm}$ ], axial turbo spin echo (TSE) T2WI (TR/TE, 1,900/76 msec; thickness, $6.0 \mathrm{~mm}$; interaction gap, $1 \mathrm{~mm}$; field of view, $25 \mathrm{~cm}$ ), and sagittal fat-suppression T2WI (TR/TE, 263/4.76 msec; thickness, $6.0 \mathrm{~mm}$; interaction gap, $1 \mathrm{~mm}$; field of view, $25 \mathrm{~cm}$ ) were obtained. The axial, sagittal and coronal T1WI with fat saturation after gadolinium diethylenetriamine pentaacetic acid (Magnevist; Bayer, Shanghai, China) administration was performed on 4 patients using the parameters used in pre-contrast T1WI.

Image analysis. The CT and MRI examinations were reviewed in conference by 2 experienced radiologists. The following features of ovarian lesions were noted: The location (left or right side), size (mean diameter of the three planes), shape (round, oval or lobulated), boundary (clear or obscure), components (solid, cystic or mixed), CT density,
MRI signal intensity, and ascites in the abdomen and pelvis. The tumors were graded into 1 of 5 degrees, depending on the solid and cystic portion in the mass: Degree 1, pure solid (no radiological evidence of cysts or necrosis); degree 2 , predominantly solid (cystic portion $<25 \%$ ); degree 3 , mixed solid and cystic (cystic portion 25-50\%); degree 4 , predominantly cystic (cystic portion $51-75 \%$ ); and degree 5, cystic (cystic portion 76-100\%). The enhancement degree of the solid components within tumors was compared with that of the uterine myometrium. The MR signal intensity of the tumor was compared with that of the adjacent pelvic skeletal muscle.

Pathological examination. Surgical resections were performed in all 25 cases. Pathological specimens were fixed in $4 \%$ formaldehyde ( $24 \mathrm{~h}$ at room temperature), embedded in paraffin and cut into sections ( $4-\mu \mathrm{m}$ thick), and were subsequently stained with hematoxylin ( $8 \mathrm{~min}$ at room temperature) and eosin (10 sec at room temperature) solution. In addition, immunohistochemical staining was performed on 9 tumor samples. The following primary antibodies were used: Cytokeratin (CK)-7 (dilution, 1:80; catalog no. OV-TL 12/30); vimentin (dilution, 1:20; catalog no. V9); epithelial membrane antigen (EMA; dilution, 1:50; catalog no. E29); Ki-67 (dilution, 1:100; catalog no. MIB-1); (Dako; Agilent Technologies, Inc., Santa Clara, CA, USA); CK8 (dilution, 1:100; catalog no. ab53280); CK17 (dilution, 1:20; catalog no. ab109725); anti-inhibin (dilution, 1:20; catalog no. ab14087); (Abcam, Cambridge, UK.). The expression of Ki-67, vimentin, inhibin-a and SMA was detected.

Statistical analysis. Statistical analysis was performed using SPSS 17.0 software (SPSS, Inc., Chicago, IL, USA). Qualitative data are expressed as percentages. Quantitative data are shown as mean \pm standard deviation. Spearman's rank correlation analysis was used to determine the correlation between the tumor size and the grade of tumor and ascites. $\mathrm{P}<0.05$ was deemed to indicate statistical significance.

\section{Results}

Clinical data. The clinical data are shown in Table I. The age of the patients ranged between 41 and 82 years (mean, $60.7 \pm 10.8$ years). A total of $23(92 \%)$ patients were postmenopausal and $2(8 \%)$ were premenopausal. Pelvic pain $(n=7)$, postmenopausal vaginal bleeding $(n=5)$ and a pelvic mass $(n=9)$ found by routine ultrasound examination were the main complaints reported by patients. There were 4 asymptomatic cases. Carbohydrate antigen 125 (CA125; normal reference, $\leq 35 \mathrm{U} / \mathrm{ml}$ ) testing was performed on 9 patients and abnormal values were observed in 6 patients.

$C T$ and MRI findings. The imaging features are described in Table II. All 25 tumors were unilateral; 10 lesions (40\%) were on the left side and $15(60 \%)$ were on the right. A total of 24 tumors $(96 \%)$ had well-defined boundaries, whereas $1(4 \%)$ tumor had an obscure boundary. In total, 15 masses were oval or round and 10 masses were lobulated. The size of these masses ranged between 1.5 and $21.4 \mathrm{~cm}$, with a mean diameter of $9.8 \pm 5.3 \mathrm{~cm} ; 18$ masses $(72 \%)$ were $>6.0 \mathrm{~cm}$ in size. 
Table I. Clinical data of patients.

\begin{tabular}{|c|c|}
\hline Clinical data & Value \\
\hline $\mathrm{Age}^{\mathrm{a}}$ (range), years & $60.7 \pm 10.8(41-82)$ \\
\hline \multicolumn{2}{|l|}{ Menopause, n (\%) } \\
\hline Premenopausal & $2(8.0)$ \\
\hline Postmenopausal & $23(92.0)$ \\
\hline \multicolumn{2}{|l|}{ Side, n (\%) } \\
\hline Left & $10(40.0)$ \\
\hline Right & $15(60.0)$ \\
\hline \multicolumn{2}{|l|}{ Pelvic pain, n (\%) } \\
\hline Yes & $7(28.0)$ \\
\hline No & $18(72.0)$ \\
\hline \multicolumn{2}{|c|}{ Postmenopausal vaginal bleeding, n (\%) } \\
\hline Yes & $5(20.0)$ \\
\hline No & $20(80.0)$ \\
\hline \multicolumn{2}{|l|}{ Palpable mass, n (\%) } \\
\hline Yes & $9(36.0)$ \\
\hline No & $16(64.0)$ \\
\hline Asymptomatic, n (\%) & $4(16.0)$ \\
\hline Tumor size $^{\mathrm{a}}$ (range), $\mathrm{cm}$ & $9.8 \pm 5.3(1.5-21.4)$ \\
\hline$\geq 6.0, \mathrm{n}(\%)$ & $18(72.0)$ \\
\hline$<6.0, \mathrm{n}(\%)$ & $7(28.0)$ \\
\hline \multicolumn{2}{|l|}{ Tumor nature, n (\%) } \\
\hline Benign & $24(96.0)$ \\
\hline Malignant & $1(4.0)$ \\
\hline
\end{tabular}

aData are shown as mean \pm standard deviation.

Solid and predominantly solid masses were found in 16 cases: 6 cases were degree 1, 4 cases were degree 2 and 6 cases were degree 3 . Cystic and predominantly cystic masses were found in 9 cases, and of these, 4 cases were degree 4 and 5 cases were degree 5 .

The CT value of the solid regions of these masses ranged between 31 and $79 \mathrm{HU}$, with a mean of $44.2 \pm 12.8 \mathrm{HU}$. MRI could clearly reveal the solid and cystic components of these tumors. The solid regions of these tumors exhibited isointensity or slight hypointensity relative to the adjacent myometrium on T1WI and T2WI. Edema or cystic regions of tumors exhibted hypointensity on T1WI and hyperintensity on T2WI (Fig. 1). Contrast-enhanced CT or MRI scans were performed on 19 patients, solid components exhibited mild to moderate enhancement in 18 cases $(94.7 \%)$ and 1 case $(5.3 \%)$ exhibited intense enhancement. The enhancement degree of these mild to moderately enhanced lesions was lower than that of normal myometrium on CT and MRI images (Figs. 1-3). The time-density curve of the tumor plateaued, following an initial mild enhancement (Fig. 4).

More detailed information was also obtained about the surrounding organs, including the uterus, ovaries and bowels. Thickened endometrium (Figs. 1 and 2) and ascites (Figs. 1, 2 and 5) were found in 7 and 9 patients, respectively. No patients exhibited an enlarged lymph node. No calcification was observed in any tumor. In addition, the
Table II. Summary of CT/MRI findings.

\begin{tabular}{|c|c|}
\hline Variable & Value \\
\hline \multicolumn{2}{|l|}{ Shape, n (\%) } \\
\hline Oval/round & $15(60.0)$ \\
\hline Lobulated & $10(40.0)$ \\
\hline \multicolumn{2}{|l|}{ Boundary, n (\%) } \\
\hline Clear & $24(96.0)$ \\
\hline Obscure & $1(4.0)$ \\
\hline \multicolumn{2}{|l|}{ Solid and cystic pattern, n (\%) } \\
\hline \multicolumn{2}{|l|}{ Solid and $50 \%$ solid } \\
\hline Degree 1 & $6(24.0)$ \\
\hline Degree 2 & $4(16.0)$ \\
\hline Degree 3 & $6(24.0)$ \\
\hline \multicolumn{2}{|l|}{ Cystic and $50 \%$ solid } \\
\hline Degree 4 & $4(16.0)$ \\
\hline Degree 4 & $5(20.0)$ \\
\hline Endometrial hyperplasia, n (\%) & $7(28.0)$ \\
\hline Ascites, $\mathrm{n}(\%)$ & $9(36.0)$ \\
\hline $\begin{array}{l}\text { Unenhanced CT attenuation of } \\
\text { tumor parenchyma }{ }^{\mathrm{a}} \text { (range), } \mathrm{HU}\end{array}$ & $44.2 \pm 12.8(31.0-79.0)$ \\
\hline \multicolumn{2}{|l|}{$\begin{array}{l}\text { MRI signal of tumor } \\
\text { parenchyma, } n(\%)\end{array}$} \\
\hline \multicolumn{2}{|l|}{ TIWI intensity } \\
\hline Iso- or mild hypointensity & $5(100)$ \\
\hline Hyperintensity & 0 \\
\hline \multicolumn{2}{|l|}{ T2WI intensity } \\
\hline Iso- or mild hypointensity & $3(60.0)$ \\
\hline Mainly isointensity & $2(40.0)$ \\
\hline Hyperintensity & 0 \\
\hline \multicolumn{2}{|l|}{$\begin{array}{l}\mathrm{CT} \text { and MRI enhancement of } \\
\text { tumor parenchyma, } \mathrm{n}(\%)\end{array}$} \\
\hline Mild & $14(73.7)$ \\
\hline Moderate & $4(21.1)$ \\
\hline Marked & $1(5.3)$ \\
\hline
\end{tabular}

${ }^{\text {aData }}$ are shown as mean \pm standard deviation. CT, computed, tomography; MRI, magnetic resonance imaging.

tumor size was significantly correlated with tumor cystic degeneration $(r=0.77, \mathrm{P}<0.001)$ and ascites $(r=0.41, \mathrm{P}<0.001)$.

Pathological examination. The tumors were oval, round or lobulated in shape. In 2 patients, the tumors were solid masses, whereas the other 23 tumors were solid-cystic masses with different proportions of solid and cystic regions. These manifestations matched the findings of the imaging. Histopathological examination revealed that the tumors were composed of varying amounts of spindle-shaped cells and theca cells, with abundant lipid and fibromatous components in the cytoplasm (Fig. 6A). A total of 19 cases were diagnosed as fibroma-thecoma and 6 were diagnosed as thecoma; 24 (96\%) tumors were benign and $1(4 \%)$ was confirmed as a low-grade malignancy. Immunohistochemical staining was performed on 9 tumors. Histologically, 7, 7, 5 and 4 tumors positively stained 

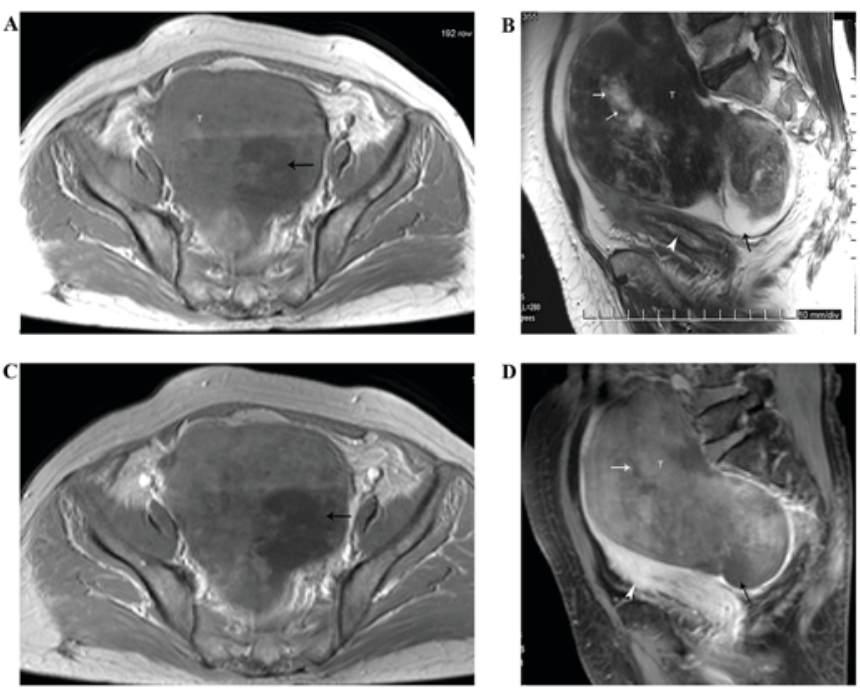

Figure 1. Right ovarian fibrothecoma in a 74-year-old woman. (A) Axial T1WI and (B) saggital T2WI magnetic resonance imaging, demonstrating a large predominant solid mass with cystic degeneration. The tumor exhibits homogeneous isointensity on T1WI and slight heterogeneous isointensity, with few patchy hyperintensity areas (black arrow) on the T2WI. The uterus is atrophied, but the thickened endometrium (arrowhead) is demonstrated on the saggital T2WI and the enhanced saggital T1WI. The solid portion of the mass exhibits moderate enhancement on (C) axial contrast-enhanced T1WI and (D) fat-suppressed T1WI. The cystic and edema areas of tumor exhibit no enhancement, whereas the myometrium (arrowhead) exhibits marked enhancement. T1WI, T1-weighted image; T, tumor.
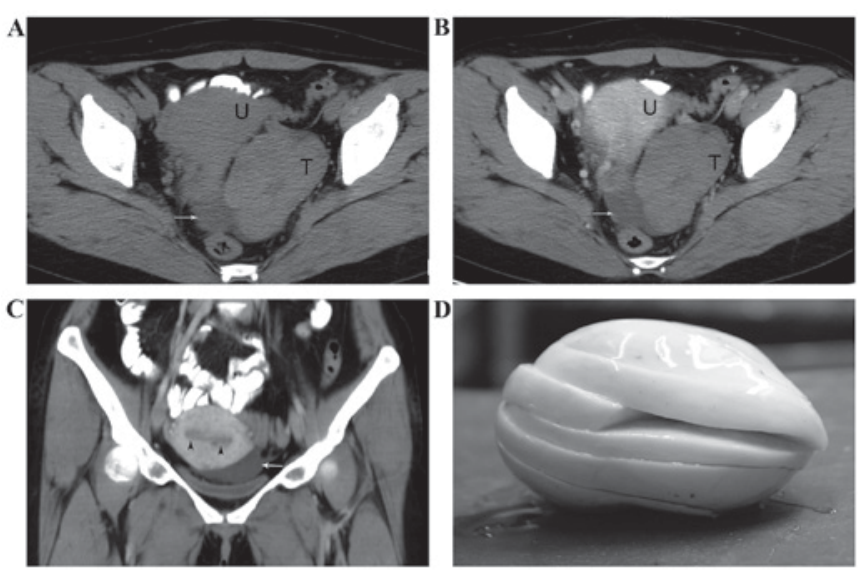

Figure 2. Left ovarian fibrothecoma in a 56-year-old woman (A) Non-contrast-enhanced axial CT image showing a large solid mass in the left side of the uterus. (B) Tumor parenchyma exhibiting mild enhancement in a contrast-enhanced CT image compared with the obviously enhanced uterus myometrium. Pelvic fluid accumulation is also revealed (white arrow). (C) Coronal image, revealing an enlarged uterus and thickened endometrium (arrowheads). (D) A macroscopic specimen, revealing that the tumor surface has a yellowish white appearance. CT, computed tomography; T, tumor; $\mathrm{U}$, uterus.

for Ki-67 (Fig. 6B), vimentin (Fig. 6C), inhibin-a (Fig. 6D) and SMA (Fig. 6E), respectively.

\section{Discussion}

Ovarian fibrothecomas are rare tumors of sex cord-stromal origin that represent $<4 \%$ of all ovarian neoplasms (4). The majority of fibrothecomas are benign and malignancy
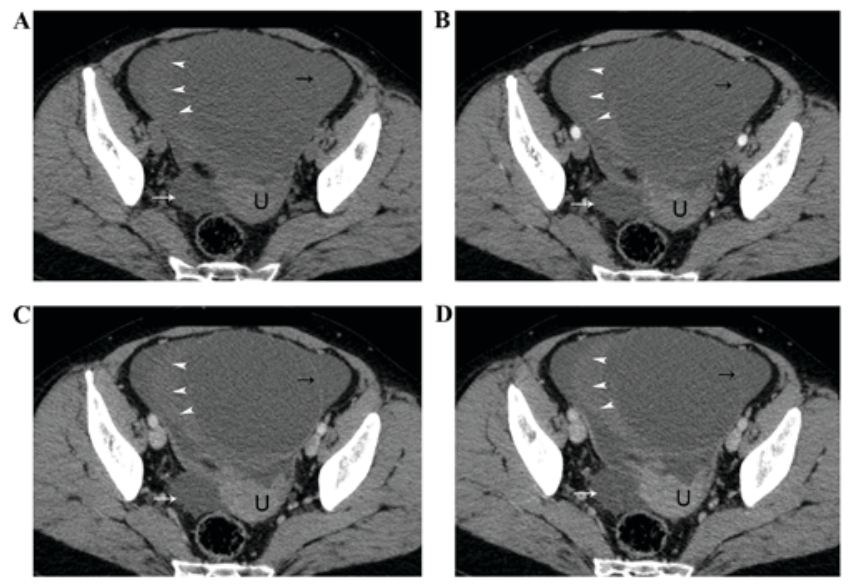

Figure 3. Right ovarian fibrothecoma with large cystic degeneration in an 82-year-old woman. (A) Axial pre-contrast CT image, revealing a lobulated, predominantly cystic tumor (cystic-solid mass) with several intratumoral solid regions (arrowheads) and linear septa (black arrow). Fluid accumulation (white arrow) exhibits a low density. Axial contrast-enhanced CT image reveals a slight enhanced tumor in (B) the arterial phase, (C) the venous phase and (D) the delay phase. The contrast of the uterus appears to be evidently enhanced. CT, computed tomography; $\mathrm{U}$, uterus.

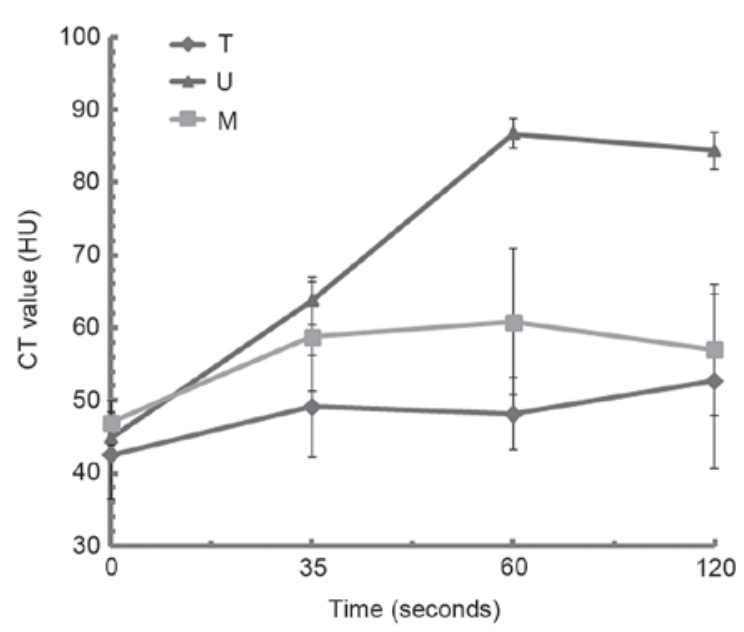

Figure 4. Time-intensity curve of ovarian fibrothecoma. The CT values of tumors, uterus and muscle, of the pelvis were $42.5 \pm 6.7,45.9 \pm 2.9$ and $46.9 \pm 2.7 \mathrm{HU}$, respectively, on pre-contrast CT images. The contrast of the tumor is mildly enhanced in the arterial phase, the time-intensity curve then plateaus in the venous and delayed phases. The tumor enhancement value is lower than that of the uterus or the muscle in all three phases. CT, computed tomography; T, tumor; $\mathrm{U}$, uterus; $\mathrm{M}$, muscle.

is rare (3). The majority of patients who suffer from this disease are postmenopausal (9). Generally, pelvic pain or distention and irregular vaginal bleeding are the main patient symptoms $(3,5,7)$. Estrogenic effects, such as endometrial hyperplasia, endometrial cancer and postmenopausal bleeding, commonly accompany fibrothecomas. In the present study, the mean patient age was 61 years and 23/25 patients were postmenopausal. A total of 5 patients manifested postmenopausal vaginal bleeding and 7 patients exhibited endometrial abnormalities. It has been reported that theca cells are endocrine cells located in the ovarian medulla (10-12). Thus, hormones secreted by theca cells are believed to be the reason for the prevalence of estrogenic activity in patients 

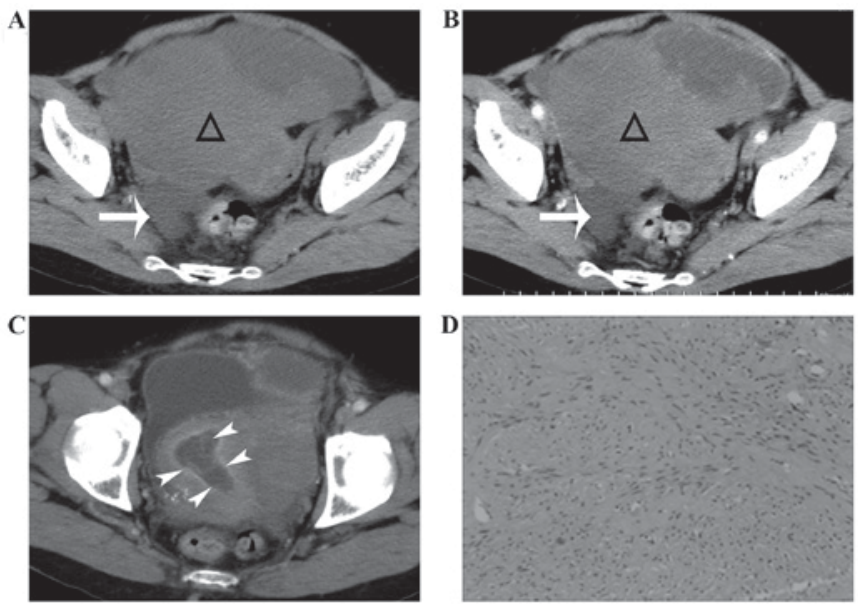

Figure 5. Left ovarian fibrothecoma in a 71-year-old woman. (A) Pre-contras CT image, revealing a large, predominantly solid mass (triangle) with peripheral cystic areas. There is also some pelvic fluid accumulation (white arrow). (B) Contrast-enhanced CT imaging revealing that the parenchyma is mildly enhanced (triangle) and the cystic component is not. (C) Substantially thickened endometrium (arrowheads) can be observed. (D) Hematoxylin and eosin staining (magnification, x100), showing the number of theca cells and spindle-shaped cells. CT, computed tomography.
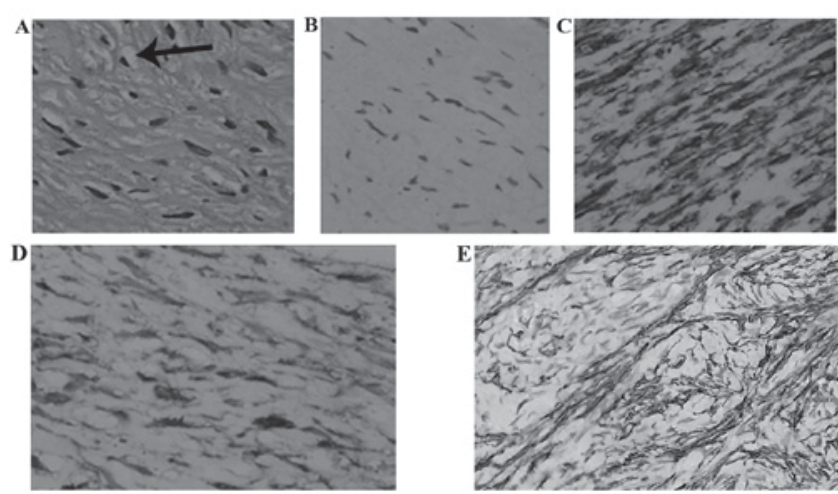

Figure 6. Histological features of fibrothecoma. (A) Hematoxylin and eosin staining (magnification, x100), revealing bundles of spindle-shaped cells and several theca cells with abundant lipid (arrow) in their cytoplasm. Immunochemical examination showed that the tumor was positive for (B) Ki-67 (5\%) (magnification, x100), (C) vimentin (magnification, $x 400$ ) and (D) inhibin-a (magnification, x100). (E) SMA (magnification, x400).

with fibrothecomas. These findings suggested that the onset age and clinical manifestations may have potential diagnostic value.

Previous studies have included as many cases as possible to achieve a comprehensive imaging view on fibrothecomas (1-4). Typically, the tumor parenchyma exhibits isodensity on CT images (1), slight hypointensity or isointensity in MRI and mild enhancement following the injection of contrast medium $(2,4)$. A thickened myometrium is usually observed in postmenopausal women during radiological examinations owing to the high level of estrogen (1-4). In the present series, the majority of the imaging features were consistent with previous studies. One important feature of fibrothecomas is the lack of blood supply (1). Ultrasound examinations have also shown that tumors lack blood flow (4). The tumors exhibit heterogeneous hypodensity or isodensity on CT imaging and
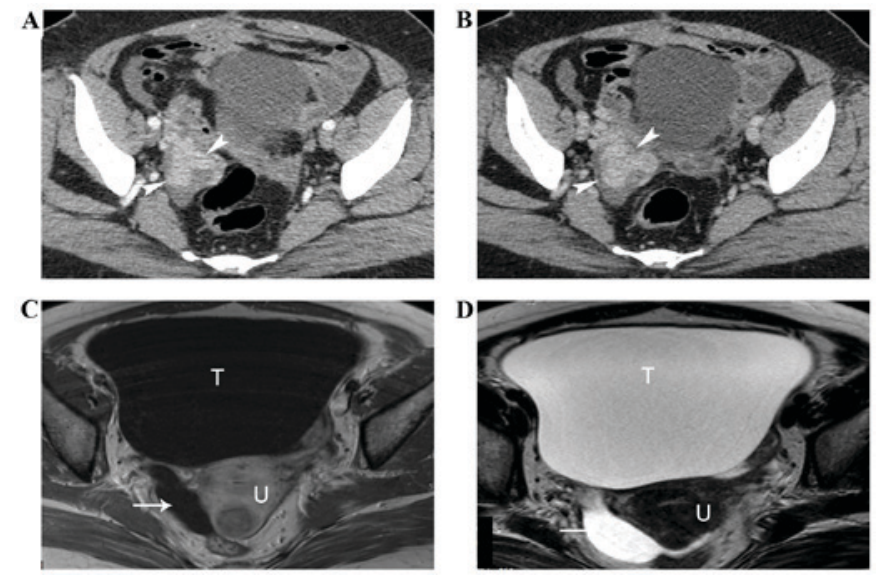

Figure 7. Atypical manifestation of a fibrothecoma in a 71-year-old (A,B) woman and a 65-year-old woman (C,D). (A and B) Contrast-enhanced CT scans revealing a solid tumor with vivid enhancement (arrowheads), which was misdiagnosed as a sclerosing stroma tumor. Histological examination revealed a thecoma with an actively growing pattern. (C and D) MRI, revealing a purely cystic fibrothecoma with a few pelvic fluid accumulations (arrow in C). There was no solid component in this tumor, which was misdiagnosed as a serous cystadenoma. CT, computed tomography; MRI, magnetic resonance imaging; $\mathrm{T}$, tumor; $\mathrm{U}$, uterus.

the tumor parenchyma shows mild to moderate enhancement after contrast medium is injected; marked enhancement is rare. Typically, the time-density curve of the tumor reveals a plateaued pattern with little initial contrast-medium uptake. This type of time-density curve is primarily observed in tumors with abundant fibrosis. The parenchyma of fibrothecomas typically exhibits hypointensity to isointensity on T1WI and T2WI when compared with myometrium. Abundant collagen, fibrostic content and the low blood flow of the tumor are believed to be the reasons that these features are observed on CT and MRI scans.

Further disease manifestations were reported in the present series. The current study showed a significant positive correlation between the tumor size and the degree of cystic degeneration. This result also suggests that fibrothecomas are a type of hypovascular tumor and that the central necrosis is partly caused by lack of blood supply, which was consistent with a previous study (13). In the present study, there were $19(76 \%)$ masses exhibiting cystic degeneration, which is a slightly higher than that observed in previous studies $(5,14)$. Only fibrothecomas were included in the present study, which may manifest as larger masses; cystic degeneration or necrosis is more frequent in fibrothecomas than fibromas (14).

Fibrothecomas can present with ascites and pleural effusion, known as Meigs' syndrome $(2,8)$. In the present study, 10 (40\%) patients exhibited ascites, which is close to the values reported in previous studies $(1,4)$. No pleural effusion occurred in the present 25 patients. Zhang et al (1) reported that larger tumors tended to be associated with ascites. In the present study, ascites was also positively associated with tumor size. An ovarian neoplasm accompanied by ascites may lead to a misdiagnosis as a malignant tumor, such as cystadenocarcinoma. The solid region and the septum of cystadenocarcinomas typically exhibit vivid enhancement, which is higher than that observed in fibrothecomas (15). Thus, when a large ovarian tumor is found with pelvic ascites and the solid region of the 
tumor exhibits mild to moderate enhancement, fibrothecoma should be considered as a potential diagnosis.

Histologically, fibrothecomas are composed of ovoid, spindle-shaped or plump tumor cells with round to ovoid-shaped nuclei and abundant intracytoplasmic lipids (1,2,5). Immunohistochemical staining reveals that the majority of fibrothecomas are positive for inhibin-a, vimentin, Ki-67 and SMA $(1,3,8)$. The present results are consistent with those of previous reports. In a total of 9 patients, inhibin-a and SMA staining was observed in 5 and 4 patients, respectively. Inhibin is an endocrine hormone produced by granulosa cells and testicular Sertoli cells (16). Laboratory tests also indicated that CA125 levels were higher than normal in $67 \%$ of patients (6/9). These are considered to be important markers for the diagnosis of sex cord-stromal tumors, particularly fibrothecomas. Additionally, 7/9 patients were positive for vimentin and $\mathrm{Ki}-67$ expression. Vimentin is a type of intermediate filament protein expressed in mesenchymal cells, whereas $\mathrm{Ki}-67$ is involved in cell proliferation and is only expressed in the active stage of the cell cycle (17). In the present study, the tumor of 1 patient exhibited vivid enhancement on CT scans (Fig. 7A and B); immunohistochemical examination showed that the tumor was positive for Ki-67 expression. Expression of Ki-67 may indicate that the tumor is actively growing. Further study is necessary to confirm whether this expression is correlated with the atypical intense enhancement of the tumor parenchyma.

There is overlap between fibrothecomas and other types of tumors, including subserous leiomyomas, fibromas, Brenner tumors and cystadenomas. Thus, a differential diagnosis is required for accurate diagnosis. Subserous leiomyomas commonly appear as solid masses, with or without necrosis, similar to solid or predominant solid fibrothecomas. However, leiomyomas have a rich vascular supply (3). Thus, enhanced CT or MRI examinations are important. Fibromas and fibrothecomas are all sex-cord stromal tumors with broadly similar appearances (18). Similar to fibrothecomas, fibromas are a type of hypovascular tumor; however, they exhibit less necrosis and cystic degeneration (19). In addition, the estrogen levels and the endometrium of patients with fibromas are always normal (20). In the present study, 8 atypical cases appeared as cystic masses (Fig. 7C and D), with few solid components. They could easily have been misdiagnosed as Brenner tumors or cystadenomas, depending on the imaging features. Low signal intensities on T2WI are also observed in Brenner tumors (21). Brenner tumors are commonly associated with ipsilateral ovarian neoplasms (22), which are hard to differentiate from cystic and predominant cystic fibrothecomas (23). Brenner tumors are usually observed in premenopausal women (21), whereas the majority of fibrothecomas are found in postmenopausal women; patients with fibrothecomas can incur higher levels of estrogen. The parenchyma or septa of cystadenomas exhibit intense enhancement after administration of contrast medium (23), whereas no such character is observed in fibrothecomas. The vividly enhanced fibrothecoma should be differentiated from a sclerosing stromal tumor (SST) of the ovary, as SSTs are usually found in younger women (19).

There are several limitations to the present study. Firstly, the number of cases is inadequate; there may be certain other features of this tumor that have not been observed owing to the small sample size. Secondly, contrast enhancement was not performed in all the patients, and further investigations are therefore required to show the enhancing features. Finally, estrogen levels were not tested in these patients.

In summary, fibrothecomas exhibit various imaging findings. Typically, they are well-defined tumors that occur in postmenopausal woman. The tumor parenchyma exhibits isodensity on CT scans, a hypointensity or isointensity signal on T1WI and T2WI, and mild to moderate enhancement after the administration of contrast medium. When plotted, the time-density curve for the tumor forms a plateau. Tumor sizes are positively correlated with cystic degeneration and pelvic ascites. The presence of estrogenic effects, such as endometrial hyperplasia, postmenopausal bleeding, elevated serum CA125 levels and positive expression of inhibin-a and Ki-67, are valuable for fibrothecoma diagnosis.

\section{Acknowledgements}

The present study was funded by the National Natural Science Foundation of China (grant no. 81471705).

\section{References}

1. Zhang Z, Wu Y and Gao J: CT diagnosis in the thecoma-fibroma group of the ovarian stromal tumors. Cell Biochem Biophys 71: 937-943, 2015

2. Zhang H, Zhang GF, Wang TP and Zhang H: Value of $3.0 \mathrm{~T}$ diffusion-weighted imaging in discriminating thecoma and fibrothecoma from other adnexal solid masses. J Ovarian Res 6: $58,2013$.

3. Li X, Zhang W, Zhu G, Sun C, Liu Q and Shen Y: Imaging features and pathologic characteristics of ovarian thecoma. J Comput Assist Tomogr 36: 46-53, 2012.

4. Shinagare AB, Meylaerts LJ, Laury AR and Mortele KJ: MRI features of ovarian fibroma and fibrothecoma with histopathologic correlation. AJR Am J Roentgenol 198: W296-W303, 2012.

5. Yen P, Khong K, Lamba R, Corwin MT and Gerscovich EO: Ovarian fibromas and fibrothecomas: Sonographic correlation with computed tomography and magnetic resonance imaging: A 5-year single-institution experience. J Ultrasound Med 32: 13-18, 2013.

6. Björkholm E and Silfverswärd C: Theca-cell tumors. Clinical features and prognosis. Acta Radiol Oncol 19: 241-244, 1980.

7. Wu B, Peng WJ, Gu YJ, Cheng YF and Mao J: MRI diagnosis of ovarian fibrothecomas: Tumour appearances and oestrogenic effect features. Br J Radiol 87: 20130634, 2014.

8. Nocito AL, Sarancone S, Bacchi C and Tellez T: Ovarian thecoma: Clinicopathological analysis of 50 cases. Ann Diagn Pathol 12: 12-16, 2008.

9. Buy JN and Ghossain M: Sex cord-stromal tumors. In: Gynecological Imaging. Springer, Berlin, Heidelberg, pp329-375, 2013.

10. Magoffin DA: Ovarian theca cell. Int J Biochem Cell Biol 37: 1344-1349, 2005.

11. Outwater EK, Wagner BJ, Mannion C, McLarney JK and Kim B: Sex cord-stromal and steroid cell tumors of the ovary. Radiographics 18: 1523-1546, 1998.

12. Tanaka YO, Tsunoda H, Kitagawa Y, Ueno T, Yoshikawa H and Saida Y: Functioning ovarian tumors: Direct and indirect findings at MR imaging. Radiographics 24 (Suppl 1): S147-S166, 2004.

13. Kato H, Kanematsu M, Ono H, Yano R, Furui T, Morishige K and Hatano Y: Ovarian fibromas: MR imaging findings with emphasis on intratumoral cyst formation. Eur J Radiol 82: e417-e421, 2013.

14. Chung BM, Park SB, Lee JB, Park HJ, Kim YS and Oh YJ: Magnetic resonance imaging features of ovarian fibroma, fibrothecoma, and thecoma. Abdom Imaging 40: 1263-1272, 2015.

15. Malek M, Pourashraf M, Mousavi AS, Rahmani M, Ahmadinejad N, Alipour A, Hashemi FS and Shakiba M: Differentiation of benign from malignant adnexal masses by functional 3 tesla MRI techniques: Diffusion-weighted imaging and time-intensity curves of dynamic contrast-enhanced MRI. Asian Pac J Cancer Prev 16: 3407-3412, 2015. 
16. Robertson DM, Pruysers E and Jobling T: Inhibin as a diagnostic marker for ovarian cancer. Cancer Lett 249: 14-17, 2007.

17. Winking $\mathrm{H}$, Gerdes $\mathrm{J}$ and Traut $\mathrm{W}$ : Expression of the proliferation marker Ki-67 during early mouse development. Cytogenet Genome Res 105: 251-256, 2004.

18. Horta M and Cunha TM: Sex cord-stromal tumors of the ovary: A comprehensive review and update for radiologists. Diagn Interv Radiol 21: 277-286, 2015.

19. Genç M, Solak A, Genç B, Sivrikoz ON, Kurtulmuş S, Turan A, Sahin N and Gür EB: A diagnostic dilemma for solid ovarian masses: The clinical and radiological aspects with differential diagnosis of 23 cases. Eur J Gynaecol Oncol 36: 186-191, 2015.

20. Cornitescu FI, Tănase F, Simionescu C and Iliescu D Clinical, histopathological and therapeutic considerations in non-neoplastic abnormal uterine bleeding in menopause transition. Rom J Morphol Embryol 52: 759-765, 2011.
21. Outwater EK, Siegelman ES, Kim B, Chiowanich P, Blasbalg R and Kilger A: Ovarian Brenner tumors: MR imaging characteristics. Magn Reson Imaging 16: 1147-1153, 1998.

22. Arnogiannaki N, Grigoriadis C, Zygouris D, Terzakis E, Sebastiadou $\mathrm{M}$ and Tserkezoglou A: Proliferative Brenner tumor of the ovary. clinicopathological study of two cases and review of the literature. Eur J Gynaecol Oncol 32: 576-578, 2011.

23. Zhang P, Cui Y, Li W, Ren G, Chu C and Wu X: Diagnostic accuracy of diffusion-weighted imaging with conventional MR imaging for differentiating complex solid and cystic ovarian tumors at 1.5T. World J Surg Oncol 10: 237, 2012. 